\title{
Correction to: Trajectory Tracking Control for Mobile Robots Using Reinforcement Learning and PID
}

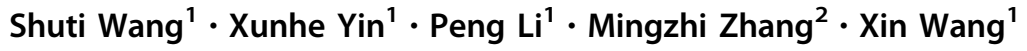

Published online: 22 January 2020

(C) Shiraz University 2020

\section{Correction to:}

Iranian Journal of Science and Technology,

Transactions of Electrical Engineering

https://doi.org/10.1007/s40998-019-00286-4

In the article "Trajectory Tracking Control for Mobile Robots Using Reinforcement Learning and PID” by Shuti Wang, Xunhe Yin, Peng Li, Mingzhi Zhang and Xin Wang
(Iranian Journal of Science and Technology, Transactions of Electrical Engineering. https://doi.org/10.1007/s40998019-00286-4), there is an error in page 5. The erratum is to correct this error.

The initial states of mobile robot are $\left[\begin{array}{lll}2.3 & -2 & 2\end{array}\right]^{T}$. We apologize for any inconvenience caused.

The original article can be found online at https:// doi.org/10.1007/s40998-019-00286-4.

\section{Xunhe Yin}

xhyin@bjtu.edu.cn

1 School of Electronic and Information Engineering, Beijing Jiaotong University, Beijing 100044, China

2 School of Electrical Engineering, Beijing Jiaotong University, Beijing 100044, China 